\title{
Smarandache Curves according to Curves on a Spacelike Surface in Minkowski 3-Space $\mathbb{R}_{1}^{3}$
}

\author{
Ufuk Ozturk and Esra Betul Koc Ozturk \\ Department of Mathematics, Faculty of Sciences, University of Çankırı Karatekin, 18100 Çankırı, Turkey \\ Correspondence should be addressed to Ufuk Ozturk; ozturkufuk06@gmail.com
}

Received 4 August 2014; Accepted 23 September 2014; Published 16 October 2014

Academic Editor: Annalisa De Bonis

Copyright (C) 2014 U. Ozturk and E. B. Koc Ozturk. This is an open access article distributed under the Creative Commons Attribution License, which permits unrestricted use, distribution, and reproduction in any medium, provided the original work is properly cited.

We introduce Smarandache curves according to the Lorentzian Darboux frame of a curve on spacelike surface in Minkowski 3space $\mathbb{R}_{1}^{3}$. Also, we obtain the Sabban frame and the geodesic curvature of the Smarandache curves and give some characterizations on the curves when the curve $\alpha$ is an asymptotic curve or a principal curve. And we give an example to illustrate these curves.

\section{Introduction}

In the theory of curves in the Euclidean and Minkowski spaces, one of the interesting problems is the characterization of a regular curve. In the solution of the problem, the curvature functions $\kappa$ and $\tau$ of a regular curve have an effective role. It is known that the shape and size of a regular curve can be determined by using its curvatures $\kappa$ and $\tau$. Another approach to the solution of the problem is to consider the relationship between the corresponding Frenet vectors of two curves. For instance, Bertrand curves and Mannheim curves arise from this relationship. Another example is the Smarandache curves. They are the objects of Smarandache geometry, that is, a geometry which has at least one Smarandachely denied axiom [1]. The axiom is said to be Smarandachely denied if it behaves in at least two different ways within the same space. Smarandache geometries are connected with the theory of relativity and the parallel universes.

By definition, if the position vector of a curve $\beta$ is composed by the Frenet frame's vectors of another curve $\alpha$, then the curve $\beta$ is called a Smarandache curve [2]. Special Smarandache curves in the Euclidean and Minkowski spaces are studied by some authors [3-8]. For instance, the special Smarandache curves according to Darboux frame in $\mathbb{E}^{3}$ are characterized in [9].

In this paper, we define Smarandache curves according to the Lorentzian Darboux frame of a curve on spacelike surface in Minkowski 3-space $\mathbb{R}_{1}^{3}$. Inspired by the previous papers we investigate the geodesic curvature and the Sabban frame's vectors of Smarandache curves. In Section 2, we explain the basic concepts of Minkowski 3-space and give Lorentzian Darboux frame that will be used throughout the paper. Section 3 is devoted to the study of four Smarandache curves, $\mathbf{T} \boldsymbol{\eta}$-Smarandache curve, $\mathbf{T} \boldsymbol{\xi}$-Smarandache curve, $\boldsymbol{\eta} \boldsymbol{\xi}$ Smarandache curve, and $\mathrm{T} \eta \xi$-Smarandache curve by considering the relationship with invariants $k_{n}, k_{g}(s)$, and $\tau_{g}(s)$ of curve on spacelike surface in Minkowski 3-space $\mathbb{R}_{1}^{3}$. Also, we give some characterizations on the curves when the curve $\alpha$ is an asymptotic curve or a principal curve. Finally, we illustrate these curves with an example.

\section{Basic Concepts}

The Minkowski 3-space $\mathbb{R}_{1}^{3}$ is the Euclidean 3-space $\mathbb{R}^{3}$ provided with the standard flat metric given by

$$
\langle\cdot, \cdot\rangle=-d x_{1}^{2}+d x_{2}^{2}+d x_{3}^{2},
$$

where $\left(x_{1}, x_{2}, x_{3}\right)$ is a rectangular Cartesian coordinate system of $\mathbb{R}_{1}^{3}$. Since $\langle\cdot, \cdot\rangle$ is an indefinite metric, recall that a nonzero vector $\mathbf{x} \in \mathbb{R}_{1}^{3}$ can have one of three Lorentzian causal characters; it can be spacelike if $\langle\mathbf{x}, \mathbf{x}\rangle>0$, timelike if $\langle\mathbf{x}, \mathbf{x}\rangle\langle 0$, and null (lightlike) if $\langle\mathbf{x}, \mathbf{x}\rangle=0$. In particular, the norm (length) of a vector $\mathbf{x} \in \mathbb{R}_{1}^{3}$ is given by $\|\mathbf{x}\|=\sqrt{|\langle\mathbf{x}, \mathbf{x}\rangle|}$ and two vectors $\mathbf{x}$ and $\mathbf{y}$ are said to be orthogonal, if $\langle\mathbf{x}, \mathbf{y}\rangle=0$. 
For any $\mathbf{x}=\left(x_{1}, x_{2}, x_{3}\right)$ and $\mathbf{y}=\left(y_{1}, y_{2}, y_{3}\right)$ in the space $\mathbb{R}_{1}^{3}$, the pseudovector product of $\mathbf{x}$ and $\mathbf{y}$ is defined by

$$
\mathbf{x} \times \mathbf{y}=\left(-x_{2} y_{3}+x_{3} y_{2}, x_{3} y_{1}-x_{1} y_{3}, x_{1} y_{2}-x_{2} y_{1}\right)
$$

Next, recall that an arbitrary curve $\alpha=\alpha(s)$ in $\mathbb{E}_{1}^{3}$ can locally be spacelike, timelike, or null (lightlike), if all of its velocity vectors $\alpha^{\prime}(s)$ are, respectively, spacelike, timelike, or null (lightlike) for every $s \in I$ [10]. If $\left\|\alpha^{\prime}(\mathrm{s})\right\| \neq 0$ for every $s \in I$, then $\alpha$ is a regular curve in $\mathbb{R}_{1}^{3}$. A spacelike (timelike) regular curve $\alpha$ is parameterized by pseudoarclength parameter $s$ which is given by $\alpha: I \subset \mathbb{R} \rightarrow$ $\mathbb{R}_{1}^{3}$; then the tangent vector $\alpha^{\prime}(s)$ along $\alpha$ has unit length; that is, $\left\langle\alpha^{\prime}(s), \alpha^{\prime}(s)\right\rangle=1\left(\left\langle\alpha^{\prime}(s), \alpha^{\prime}(s)\right\rangle=-1\right)$ for all $s \in I$, respectively.

Remark 1. Let $\mathbf{x}=\left(x_{1}, x_{2}, x_{3}\right), \mathbf{y}=\left(y_{1}, y_{2}, y_{3}\right)$, and $\mathbf{z}=$ $\left(z_{1}, z_{2}, z_{3}\right)$ be vectors in $\mathbb{R}_{1}^{3}$. Then

$$
\begin{gathered}
\text { (i) }\langle\mathbf{x} \times \mathbf{y}, \mathbf{z}\rangle=\left|\begin{array}{lll}
x_{1} & x_{2} & x_{3} \\
y_{1} & y_{2} & y_{3} \\
z_{1} & z_{2} & z_{3}
\end{array}\right|, \\
\text { (ii) } \mathbf{x} \times(\mathbf{y} \times \mathbf{z})=-\langle\mathbf{x}, \mathbf{z}\rangle \mathbf{y}+\langle\mathbf{x}, \mathbf{y}\rangle \mathbf{z}, \\
\text { (iii) }\langle\mathbf{x} \times \mathbf{y}, \mathbf{x} \times \mathbf{y}\rangle=-\langle\mathbf{x}, \mathbf{x}\rangle\langle\mathbf{y}, \mathbf{y}\rangle+\langle\mathbf{x}, \mathbf{y}\rangle^{2},
\end{gathered}
$$

where $\times$ is the pseudovector product in the space $\mathbb{R}_{1}^{3}$.

Lemma 2. In the Minkowski 3-space $\mathbb{R}_{1}^{3}$, the following properties are satisfied [10]:

(i) two timelike vectors are never orthogonal;

(ii) two null vectors are orthogonal if and only if they are linearly dependent;

(iii) timelike vector is never orthogonal to a null vector.

Let $\phi: U \subset \mathbb{R}^{2} \rightarrow \mathbb{R}_{1}^{3}, \phi(U)=M$ and $\gamma: I \subset \mathbb{R} \rightarrow U$ be a spacelike embedding and a regular curve, respectively. Then we have a curve $\alpha$ on the surface $M$ which is defined by $\alpha(s)=\phi(\gamma(s))$ and since $\phi$ is a spacelike embedding, we have a unit timelike normal vector field $\boldsymbol{\eta}$ along the surface $M$ which is defined by

$$
\boldsymbol{\eta} \equiv \frac{\phi_{x} \times \phi_{y}}{\left\|\phi_{x} \times \phi_{y}\right\|}
$$

Since $M$ is a spacelike surface, we can choose a future directed unit timelike normal vector field $\boldsymbol{\eta}$ along the surface $M$. Hence we have a pseudoorthonormal frame $\{\mathbf{T}, \boldsymbol{\eta}, \boldsymbol{\xi}\}$ which is called the Lorentzian Darboux frame along the curve $\alpha$ where $\boldsymbol{\xi}(s)=\mathbf{T}(s) \times \boldsymbol{\eta}(s)$ is a unit spacelike vector. The corresponding Frenet formulae of $\alpha$ read

$$
\left[\begin{array}{l}
\mathbf{T}^{\prime} \\
\boldsymbol{\eta}^{\prime} \\
\xi^{\prime}
\end{array}\right]=\left[\begin{array}{ccc}
0 & k_{n} & k_{g} \\
k_{n} & 0 & \tau_{g} \\
-k_{g} & \tau_{g} & 0
\end{array}\right]\left[\begin{array}{l}
\mathbf{T} \\
\boldsymbol{\eta} \\
\boldsymbol{\xi}
\end{array}\right],
$$

where $k_{n}(s)=-\left\langle\mathbf{T}^{\prime}(s), \boldsymbol{\eta}(s)\right\rangle, k_{g}(s)=\left\langle\mathbf{T}^{\prime}(s), \boldsymbol{\xi}(s)\right\rangle$, and $\tau_{g}(s)=-\left\langle\xi^{\prime}(s), \boldsymbol{\eta}(s)\right\rangle$ are the asymptotic curvature, the geodesic curvature, and the principal curvature of $\alpha$ on the surface $M$ in $\mathbb{R}_{1}^{3}$, respectively, and $s$ is arclength parameter of $\alpha$. In particular, the following relations hold:

$$
\mathrm{T} \times \boldsymbol{\eta}=\xi, \quad \eta \times \xi=-\mathrm{T}, \quad \xi \times \mathrm{T}=\boldsymbol{\eta} .
$$

Both $k_{n}$ and $k_{g}$ may be positive or negative. Specifically, $k_{n}$ is positive if $\alpha$ curves towards the normal vector $\boldsymbol{\eta}$, and $k_{g}$ is positive if $\alpha$ curves towards the tangent normal vector $\xi$. Also, the curve $\alpha$ is characterized by $k_{n}, k_{g}$, and $\tau_{g}$ as follows:

$$
\alpha \text { is } \begin{cases}\text { an asymptotic curve } & \text { iff } k_{n} \equiv 0, \\ \text { a geodesic curve } & \text { iff } k_{g} \equiv 0, \\ \text { a principal curve } & \text { iff } \tau_{g} \equiv 0 .\end{cases}
$$

Since $\alpha$ is a unit-speed curve, $\ddot{\alpha}$ is perpendicular to $\mathbf{T}$, but $\ddot{\alpha}$ may have components in the normal and tangent normal directions:

$$
\ddot{\alpha}=k_{n} \boldsymbol{\eta}+k_{g} \xi .
$$

These are related to the total curvature $\kappa$ of $\alpha$ by the formula

$$
\kappa^{2}=\|\ddot{\alpha}\|^{2}=k_{g}^{2}-k_{n}^{2} .
$$

From (9) we can give the following proposition.

Proposition 3. Let $M$ be a spacelike surface in $\mathbb{R}_{1}^{3}$. Let $\alpha=$ $\alpha(s)$ be regular unit speed curves lying fully with the Lorentzian Darboux frame $\{\mathbf{T}, \boldsymbol{\eta}, \boldsymbol{\xi}\}$ on the surface $M$ in $\mathbb{R}_{1}^{3}$. There is not a geodesic curve on $M$.

The pseudosphere with center at the origin and of radius $r=1$ in the Minkowski 3-space $\mathbb{R}_{1}^{3}$ is a quadric defined by

$$
S_{1}^{2}=\left\{\vec{x} \in \mathbb{R}_{1}^{3} \mid-x_{1}^{2}+x_{2}^{2}+x_{3}^{2}=1\right\} .
$$

Let $\beta: I \subset \mathbb{R} \rightarrow S_{1}^{2}$ be a curve lying fully in pseudosphere $S_{1}^{2}$ in $\mathbb{R}_{1}^{3}$. Then its position vector $\beta$ is a spacelike, which means that the tangent vector $T_{\beta}=\beta^{\prime}$ can be spacelike, timelike, or null. Depending on the causal character of $T_{\beta}$, we distinguish the following three cases [5].

Case 1 ( $T_{\beta}$ is a unit spacelike vector). Then we have orthonormal Sabban frame $\left\{\beta(s), T_{\beta}(s), \xi_{\beta}(s)\right\}$ along the curve $\alpha$, where $\xi_{\beta}(s)=-\beta(s) \times T_{\beta}(s)$ is the unit timelike vector. The corresponding Frenet formulae of $\beta$ according to the Sabban frame read

$$
\left[\begin{array}{c}
\beta^{\prime} \\
T_{\beta}^{\prime} \\
\xi_{\beta}^{\prime}
\end{array}\right]=\left[\begin{array}{ccc}
0 & 1 & 0 \\
-1 & 0 & -\bar{k}_{g}(s) \\
0 & -\bar{k}_{g}(s) & 0
\end{array}\right]\left[\begin{array}{c}
\beta \\
T_{\beta} \\
\xi_{\beta}
\end{array}\right],
$$

where $\bar{k}_{g}(s)=\operatorname{det}\left(\beta(s), T_{\beta}(s), T_{\beta}^{\prime}(s)\right)$ is the geodesic curvature of $\beta$ and $s$ is the arclength parameter of $\beta$. In particular, the following relations hold:

$$
\beta \times T_{\beta}=-\xi_{\beta}, \quad T_{\beta} \times \xi_{\beta}=\beta, \quad \xi_{\beta} \times \beta=T_{\beta} .
$$


Case 2 ( $T_{\beta}$ is a unit timelike vector). Hence we have orthonormal Sabban frame $\left\{\beta(s), T_{\beta}(s), \xi_{\beta}(s)\right\}$ along the curve $\beta$, where $\xi_{\beta}(s)=\beta(s) \times T_{\beta}(s)$ is the unit spacelike vector. The corresponding Frenet formulae of $\beta$ according to the Sabban frame read

$$
\left[\begin{array}{c}
\beta^{\prime} \\
T_{\beta}^{\prime} \\
\xi_{\beta}^{\prime}
\end{array}\right]=\left[\begin{array}{ccc}
0 & 1 & 0 \\
1 & 0 & \bar{k}_{g}(s) \\
0 & \bar{k}_{g}(s) & 0
\end{array}\right]\left[\begin{array}{c}
\beta \\
T_{\beta} \\
\xi_{\beta}
\end{array}\right],
$$

where $\bar{k}_{g}(s)=\operatorname{det}\left(\beta(s), T_{\beta}(s), T_{\beta}^{\prime}(s)\right)$ is the geodesic curvature of $\beta$ and $s$ is the arclength parameter of $\beta$. In particular, the following relations hold:

$$
\beta \times T_{\beta}=\xi_{\beta}, \quad T_{\beta} \times \xi_{\beta}=\beta, \quad \xi_{\beta} \times \beta=-T_{\beta} .
$$

Case 3 ( $T_{\beta}$ is a null vector). It is known that the only null curves lying on pseudosphere $S_{1}^{2}$ are the null straight lines, which are the null geodesics.

\section{Smarandache Curves according to Curves on a Spacelike Surface in Minkowski 3-Space $\mathbb{R}_{1}^{3}$}

In the following section, we define the Smarandache curves according to the Lorentzian Darboux frame in Minkowski 3-space. Also, we obtain the Sabban frame and the geodesic curvature of the Smarandache curves lying on pseudosphere $S_{1}^{2}$ and give some characterizations on the curves when the curve $\alpha$ is an asymptotic curve or a principal curve.

Definition 4. Let $\alpha=\alpha(s)$ be a spacelike curve lying fully on the spacelike surface $M$ in $\mathbb{R}_{1}^{3}$ with the moving Lorentzian Darboux frame $\{\mathbf{T}, \boldsymbol{\eta}, \boldsymbol{\xi}\}$. Then $\mathbf{T} \boldsymbol{\eta}$-Smarandache curve of $\alpha$ is defined by

$$
\beta\left(s^{\star}(s)\right)=\frac{1}{\sqrt{2}}(a \mathbf{T}(s)+b \boldsymbol{\eta}(s)),
$$

where $a, b \in \mathbb{R}_{0}$ and $a^{2}-b^{2}=2$.

Definition 5. Let $\alpha=\alpha(s)$ be a spacelike curve lying fully on the spacelike surface $M$ in $\mathbb{R}_{1}^{3}$ with the moving Lorentzian Darboux frame $\{\mathbf{T}, \boldsymbol{\eta}, \boldsymbol{\xi}\}$. Then $\mathbf{T} \boldsymbol{\xi}$-Smarandache curve of $\alpha$ is defined by

$$
\beta\left(s^{\star}(s)\right)=\frac{1}{\sqrt{2}}(a \mathbf{T}(s)+b \boldsymbol{\xi}(s)),
$$

where $a, b \in \mathbb{R}_{0}$ and $a^{2}+b^{2}=2$.

Definition 6. Let $\alpha=\alpha(s)$ be a spacelike curve lying fully on the spacelike surface $M$ in $\mathbb{R}_{1}^{3}$ with the moving Lorentzian Darboux frame $\{\mathbf{T}, \boldsymbol{\eta}, \boldsymbol{\xi}\}$. Then $\boldsymbol{\eta} \boldsymbol{\xi}$-Smarandache curve of $\alpha$ is defined by

$$
\beta\left(s^{\star}(s)\right)=\frac{1}{\sqrt{2}}(a \boldsymbol{\eta}(s)+b \boldsymbol{\xi}(s)),
$$

where $a, b \in \mathbb{R}_{0}$ and $b^{2}-a^{2}=2$.
Definition 7. Let $\alpha=\alpha(s)$ be a spacelike curve lying fully on the spacelike surface $M$ in $\mathbb{R}_{1}^{3}$ with the moving Lorentzian Darboux frame $\{\mathbf{T}, \boldsymbol{\eta}, \boldsymbol{\xi}\}$. Then $\mathbf{T} \boldsymbol{\eta} \boldsymbol{\xi}$-Smarandache curve of $\alpha$ is defined by

$$
\beta\left(s^{\star}(s)\right)=\frac{1}{\sqrt{3}}(a \mathbf{T}(s)+b \boldsymbol{\eta}(s)+c \boldsymbol{\xi}(s)),
$$

where $a, b \in \mathbb{R}_{0}$ and $a^{2}-b^{2}+c^{2}=3$.

Thus, there are two following cases.

Case 4 ( $\alpha$ is an asymptotic curve). Then, we have the following theorems.

Theorem 8. Let $\alpha=\alpha(s)$ be an asymptotic spacelike curve lying fully on the spacelike surface $M$ in $\mathbb{R}_{1}^{3}$ with the moving Lorentzian Darboux frame $\{\mathbf{T}, \boldsymbol{\eta}, \boldsymbol{\xi}\}$. Then

(i) if $a k_{g}+b \tau_{g} \neq 0$ for all $s$, then the Sabban frame $\left\{\beta, \mathbf{T}_{\beta}\right.$, $\boldsymbol{\xi}_{\beta}$ \} of the $\mathbf{T} \boldsymbol{\eta}$-Smarandache curve $\beta$ is given by

$$
\left[\begin{array}{c}
\beta \\
\mathbf{T}_{\beta} \\
\boldsymbol{\xi}_{\beta}
\end{array}\right]=\left[\begin{array}{ccc}
\frac{a}{\sqrt{2}} & \frac{b}{\sqrt{2}} & 0 \\
0 & 0 & \epsilon \\
\epsilon \frac{b}{\sqrt{2}} & \epsilon \frac{a}{\sqrt{2}} & 0
\end{array}\right]\left[\begin{array}{l}
\mathbf{T} \\
\boldsymbol{\eta} \\
\boldsymbol{\xi}
\end{array}\right],
$$

and the geodesic curvature $\bar{k}_{g}$ of the curve $\beta$ reads

$$
\bar{k}_{g}=\frac{a \tau_{g}-b k_{g}}{\sqrt{2}},
$$

where $\epsilon=\operatorname{sign}\left(a k_{g}+b \tau_{g}\right)$ for all $s$ and

$$
\frac{d s^{*}}{d s}=\epsilon \frac{a k_{g}+b \tau_{g}}{\sqrt{2}}
$$

(ii) if $a k_{g}+b \tau_{g}=0$ for all $s$, then the Sabban frame $\left\{\beta, \mathbf{T}_{\beta}\right.$, $\left.\boldsymbol{\xi}_{\beta}\right\}$ of the $\mathbf{T} \boldsymbol{\eta}$-Smarandache curve $\beta$ is a null geodesic.

Proof. We assume that the curve $\alpha$ is an asymptotic curve. Differentiating (15) with respect to $s$ and using (5) we obtain

$$
\begin{aligned}
\beta^{\prime} & =\frac{d \beta}{d s} \\
& =\frac{1}{\sqrt{2}}\left(a k_{g}+b \tau_{g}\right) \xi, \\
\left\langle\beta^{\prime}, \beta^{\prime}\right\rangle & =\frac{\left(a k_{g}+b \tau_{g}\right)^{2}}{2} .
\end{aligned}
$$

Then, there are two following cases.

(i) If $a k_{g}+b \tau_{g} \neq 0$ for all $s$, since $\beta^{\prime}=\left(d \beta / d s^{*}\right)\left(d s^{*} / d s\right)$, then the tangent vector $\mathbf{T}_{\beta}$ of the curve $\beta$ is a spacelike vector such that

$$
\mathbf{T}_{\beta}=\epsilon \boldsymbol{\xi}
$$


where

$$
\frac{d s^{*}}{d s}=\epsilon \frac{a k_{g}+b \tau_{g}}{\sqrt{2}} .
$$

On the other hand, from (15) and (23) it can be easily seen that

$$
\begin{aligned}
\xi_{\beta} & =-\beta \times T_{\beta} \\
& =\epsilon \frac{b}{\sqrt{2}} \mathbf{T}+\epsilon \frac{a}{\sqrt{2}} \boldsymbol{\eta}
\end{aligned}
$$

is a unit timelike vector.

Consequently, the geodesic curvature $\bar{k}_{g}$ of the curve $\beta=\beta\left(s^{*}\right)$ is given by

$$
\begin{aligned}
\bar{k}_{g} & =\operatorname{det}\left(\beta, T_{\beta}, \mathbf{T}_{\beta}^{\prime}\right) \\
& =\frac{a \tau_{g}-b k_{g}}{\sqrt{2}} .
\end{aligned}
$$

From (15), (23), and (25) we obtain the Sabban frame $\left\{\beta, \mathbf{T}_{\beta}, \boldsymbol{\xi}_{\beta}\right\}$ of $\beta$.

(ii) If $a k_{g}+b \tau_{g}=0$ for all $s$, then $\beta^{\prime}$ is null. So, the tangent vector $\mathbf{T}_{\beta}$ of the curve $\beta$ is a null vector. It is known that the only null curves lying on pseudosphere $S_{1}^{2}$ are the null straight lines, which are the null geodesics.

In the theorems which follow, in a similar way as in Theorem 8, we obtain the Sabban frame $\left\{\beta, \mathbf{T}_{\beta}, \boldsymbol{\xi}_{\beta}\right\}$ and the geodesic curvature $\bar{k}_{g}$ of a spacelike Smarandache curve. We omit the proofs of Theorems 9, 10, and 11, since they are analogous to the proof of Theorem 8 .

Theorem 9. Let $\alpha=\alpha(s)$ be an asymptotic spacelike curve lying fully on the spacelike surface $M$ in $\mathbb{R}_{1}^{3}$ with the moving Lorentzian Darboux frame $\{\mathbf{T}, \boldsymbol{\eta}, \boldsymbol{\xi}\}$. Then

(i) if $2 k_{g}^{2}-\left(b \tau_{g}\right)^{2} \neq 0$ for all $s$, then the Sabban frame $\{\beta$, $\left.\mathbf{T}_{\beta}, \boldsymbol{\xi}_{\beta}\right\}$ of the $\mathbf{T} \boldsymbol{\xi}$-Smarandache curve $\beta$ is given by

$$
\left[\begin{array}{c}
\beta \\
\mathbf{T}_{\beta} \\
\boldsymbol{\xi}_{\beta}
\end{array}\right]
$$$$
=\left[\begin{array}{ccc}
\frac{a}{\sqrt{2}} & 0 & \frac{b}{\sqrt{2}} \\
\frac{-b k_{g}}{a k_{g}} & \frac{b \tau_{g}}{\sqrt{\epsilon\left(2 k_{g}^{2}-\left(b \tau_{g}\right)^{2}\right)}} & \frac{\sqrt{\epsilon\left(2 k_{g}^{2}-\left(b \tau_{g}\right)^{2}\right)}}{\sqrt{\epsilon\left(2 k_{g}^{2}-\left(b \tau_{g}\right)^{2}\right)}} \\
\frac{b \tau_{g}}{\sqrt{\epsilon\left(2 k_{g}^{2}-\left(b \tau_{g}\right)^{2}\right)}} & \frac{-2 k_{g}}{\sqrt{\epsilon\left(2 k_{g}^{2}-\left(b \tau_{g}\right)^{2}\right)}} & \frac{a b \tau_{g}}{\sqrt{\epsilon\left(2 k_{g}^{2}-\left(b \tau_{g}\right)^{2}\right)}}
\end{array}\right]
$$$$
\times\left[\begin{array}{l}
\mathrm{T} \\
\boldsymbol{\eta} \\
\xi
\end{array}\right],
$$

and the geodesic curvature $\bar{k}_{g}$ of the curve $\beta$ reads

$$
\begin{aligned}
\bar{k}_{g}=( & \left(a^{2} b^{3} \tau_{g}^{3}-b^{4} \tau_{g}^{3}+4 b \tau_{g} k_{g}^{2}\right) k_{g}^{\prime} \\
& +\left(b^{4} \tau_{g}^{2} k_{g}-a^{2} b^{3} \tau_{g}^{2} k_{g}-4 b k_{g}^{3}\right) \tau_{g}^{\prime} \\
& +\left(2 a b^{2}+2 a b-4 a\right) \tau_{g} k_{g}^{4} \\
& \left.-\left(a b^{4}+a b^{3}\right) \tau_{g}^{3} k_{g}^{2}+a b^{4} \tau_{g}^{5}\right) \\
\times & \left(\left(4 k_{g}^{2}-2\left(b \tau_{g}\right)^{2}\right)^{2}\right)^{-1}
\end{aligned}
$$

where $\epsilon=\operatorname{sign}\left(2 k_{g}^{2}-\left(b \tau_{g}\right)^{2}\right)$ for all $s$ and

$$
\frac{d s^{*}}{d s}=\sqrt{\epsilon \frac{2 k_{g}^{2}-\left(b \tau_{g}\right)^{2}}{2}}
$$

(ii) if $a k_{g}+b \tau_{g}=0$ for all $s$, then the Sabban frame $\left\{\beta, \mathbf{T}_{\beta}\right.$, $\left.\boldsymbol{\xi}_{\beta}\right\}$ of the $\mathbf{T} \boldsymbol{\eta}$-Smarandache curve $\beta$ is a null geodesic.

Theorem 10. Let $\alpha=\alpha(s)$ be an asymptotic spacelike curve lying fully on the spacelike surface $M$ in $\mathbb{R}_{1}^{3}$ with the moving Lorentzian Darboux frame $\{\mathbf{T}, \boldsymbol{\eta}, \boldsymbol{\xi}\}$. Then

(i) if $\left(b k_{g}\right)^{2}-2 \tau_{g}^{2} \neq 0$ for all $s$, then the Sabban frame $\{\beta$, $\left.\mathbf{T}_{\beta}, \boldsymbol{\xi}_{\beta}\right\}$ of the $\boldsymbol{\eta} \boldsymbol{\xi}$-Smarandache curve $\beta$ is given by

$\left[\begin{array}{c}\beta \\ \mathbf{T}_{\beta} \\ \boldsymbol{\xi}_{\beta}\end{array}\right]$

$=\left[\begin{array}{ccc}0 & \frac{a}{\sqrt{2}} & \frac{b}{\sqrt{2}} \\ \frac{-b k_{g}}{\sqrt{\epsilon\left(\left(b k_{g}\right)^{2}-2 \tau_{g}^{2}\right)}} & \frac{b \tau_{g}}{\sqrt{\epsilon\left(\left(b k_{g}\right)^{2}-2 \tau_{g}^{2}\right)}} & \frac{a \tau_{g}}{\sqrt{\epsilon\left(\left(b k_{g}\right)^{2}-2 \tau_{g}^{2}\right)}} \\ \frac{-2 \tau_{g}}{\sqrt{2 \epsilon\left(\left(b k_{g}\right)^{2}-2 \tau_{g}^{2}\right)}} & \frac{b^{2} k_{g}}{\sqrt{2 \epsilon\left(\left(b k_{g}\right)^{2}-2 \tau_{g}^{2}\right)}} & \frac{-a b k_{g}}{\sqrt{2 \epsilon\left(\left(b k_{g}\right)^{2}-2 \tau_{g}^{2}\right)}}\end{array}\right]$ $\times\left[\begin{array}{l}\mathbf{T} \\ \eta \\ \xi\end{array}\right]$,

and the geodesic curvature $\bar{k}_{g}$ of the curve $\beta$ reads

$$
\begin{aligned}
\bar{k}_{g}=( & \left(4 \sqrt{2} b \tau_{g}^{3}-\left(\sqrt{2} a^{2} b^{3}+\sqrt{2} b^{5}\right) \tau_{g} k_{g}^{2}\right) k_{g}^{\prime} \\
& +\left(\left(\sqrt{2} a^{2} b^{3}+\sqrt{2} b^{5}\right) k_{g}^{3}-4 \sqrt{2} b \tau_{g}^{2} k_{g}\right) \tau_{g}^{\prime} \\
& -\sqrt{2} a b^{4} k_{g}^{5}+2 \sqrt{2} a b^{4} \tau_{g}^{2} k_{g}^{3} \\
& \left.+\left(4 \sqrt{2} a-4 \sqrt{2} a b^{2}\right) \tau_{g}^{4} k_{g}\right) \\
\times & \left(2\left(b^{2} k_{g}^{2}-2 \tau_{g}^{2}\right)^{2}\right)^{-1},
\end{aligned}
$$


where $\epsilon=\operatorname{sign}\left(\left(b k_{g}\right)^{2}-2 \tau_{g}^{2}\right)$ for all s and

$$
\frac{d s^{*}}{d s}=\sqrt{\epsilon \frac{\left(b k_{g}\right)^{2}-2 \tau_{g}^{2}}{2}} ;
$$

(ii) if $\left(b k_{g}\right)^{2}-2 \tau_{g}^{2}=0$ for all $s$, then the Sabban frame $\left\{\beta, \mathbf{T}_{\beta}, \boldsymbol{\xi}_{\beta}\right\}$ of the $\mathbf{T} \boldsymbol{\eta}$-Smarandache curve $\beta$ is a null geodesic.

Theorem 11. Let $\alpha=\alpha(s)$ be an asymptotic spacelike curve lying fully on the spacelike surface $M$ in $\mathbb{R}_{1}^{3}$ with the moving Lorentzian Darboux frame $\{\mathbf{T}, \boldsymbol{\eta}, \boldsymbol{\xi}\}$. Then

(i) if $3\left(k_{g}^{2}-\tau_{g}^{2}\right)+\left(b k_{g}+a \tau_{g}\right)^{2} \neq 0$ for all s, then the Sabban frame $\left\{\beta, \mathbf{T}_{\beta}, \boldsymbol{\xi}_{\beta}\right\}$ of the $\mathbf{T} \boldsymbol{\eta} \boldsymbol{\xi}$-Smarandache curve $\beta$ is given by

$$
\begin{gathered}
\beta=\frac{a \mathbf{T}+b \boldsymbol{\eta}+c \boldsymbol{\xi}}{\sqrt{3}}, \\
\mathbf{T}_{\beta}=\frac{-c k_{g} \mathbf{T}+c \tau_{g} \boldsymbol{\eta}+\left(a k_{g}+b \tau_{g}\right) \boldsymbol{\xi}}{\sqrt{\epsilon\left(3\left(k_{g}^{2}-\tau_{g}^{2}\right)+\left(b k_{g}+a \tau_{g}\right)^{2}\right)}}, \\
\xi_{\beta}=\left(\left(b\left(a k_{g}+b \tau_{g}\right)-c^{2} \tau_{g}\right) \mathbf{T}\right. \\
\left.+\left(c^{2} k_{g}+a\left(a k_{g}+b \tau_{g}\right)\right) \boldsymbol{\eta}-\left(a c \tau_{g}+b c k_{g}\right) \boldsymbol{\xi}\right) \\
\times\left(\sqrt{\epsilon\left(9\left(k_{g}^{2}-\tau_{g}^{2}\right)+3\left(b k_{g}+a \tau_{g}\right)^{2}\right)}\right)^{-1},
\end{gathered}
$$

and the geodesic curvature $\bar{k}_{g}$ of the curve $\beta$ reads

$$
\begin{aligned}
\bar{k}_{g}= & \left(\left(b\left(a k_{g}+b \tau_{g}\right)-c^{2} \tau_{g}\right) f_{1}\right. \\
& -\left(c^{2} k_{g}+a\left(a k_{g}+b \tau_{g}\right)\right) f_{2} \\
& \left.-\left(a c \tau_{g}+b c k_{g}\right) f_{3}\right) \\
\times & \left(\sqrt{3}\left(3\left(k_{g}^{2}-\tau_{g}^{2}\right)+\left(b k_{g}+a \tau_{g}\right)^{2}\right)^{2}\right)^{-1},
\end{aligned}
$$

where $\epsilon=\operatorname{sign}\left(3\left(k_{g}^{2}-\tau_{g}^{2}\right)+\left(b k_{g}+a \tau_{g}\right)^{2}\right)$ for all $s$ and

$$
\begin{aligned}
& \frac{d s^{*}}{d s}=\sqrt{\epsilon \frac{\left(b k_{g}\right)^{2}-2 \tau_{g}^{2}}{2}} \\
f_{1}= & \left(\left(a^{2} c-3 c\right) \tau_{g}^{2}+a b c \tau_{g} k_{g}\right) k_{g}^{\prime} \\
+ & \left(\left(3 c-a^{2} c\right) \tau_{g} k_{g}-a b c k_{g}^{2}\right) \tau_{g}^{\prime} \\
+ & \left(3 a+a b^{2}\right) k_{g}^{4}+\left(a^{3}+2 a b^{2}-3 a\right) \tau_{g}^{2} k_{g}^{2} \\
+ & \left(b^{3}+2 a^{2} b+3 b\right) \tau_{g} k_{g}^{3} \\
+ & \left(a^{2} b-3 b\right) \tau_{g}^{3} k_{g},
\end{aligned}
$$

$$
\begin{gathered}
f_{2}=\left(a b c \tau_{g}^{2}+\left(b^{2} c+3 c\right) \tau_{g} k_{g}\right) k_{g}^{\prime} \\
-\left(a b c \tau_{g} k_{g}+\left(3 c+b^{2} c\right) k_{g}^{2}\right) \tau_{g}^{\prime} \\
+\left(3 b-a^{2} b\right) \tau_{g}^{4}-\left(b^{3}+2 a^{2} b+3 b\right) \tau_{g}^{2} k_{g}^{2} \\
-\left(a b^{2}+3 a\right) \tau_{g} k_{g}^{3} \\
+\left(3 a-2 a b^{2}-a^{3}\right) \tau_{g}^{3} k_{g}, \\
f_{3}=\left(\left(-a^{3}+3 a+a b^{2}\right) \tau_{g}^{2}+\left(b^{3}+3 b-a^{2} b\right) \tau_{g} k_{g}\right) k_{g}^{\prime} \\
+\left(\left(-b^{3}-3 b+a^{2} b\right) k_{g}^{2}+\left(a^{3}-a b^{2}-3 a\right) \tau_{g} k_{g}\right) \tau_{g}^{\prime} \\
+\left(3 c+b^{2} c\right) k_{g}^{4}+\left(3 c-a^{2} c\right) \tau_{g}^{4}+2 a b c \tau_{g} k_{g}^{3} \\
-2 a b c \tau_{g}^{3} k_{g}+\left(a^{2} c-6 c-b^{2} c\right) \tau_{g}^{2} k_{g}^{2} ; \\
\text { (ii) if } 3\left(k_{g}^{2}-\tau_{g}^{2}\right)+\left(b k_{g}+a \tau_{g}\right)^{2}=0 \text { for alls, then the Sabban } \\
\text { frame }\left\{\beta, \mathbf{T}_{\beta}, \xi_{\beta}\right\} \text { of the Ty-Smarandache curve } \beta \text { is a } \\
\text { null geodesic. }
\end{gathered}
$$

Case 5 ( $\alpha$ is a principal curve). Then, we have the following theorems.

Theorem 12. Let $\alpha=\alpha(s)$ be a principal spacelike curve lying fully on the spacelike surface $M$ in $\mathbb{R}_{1}^{3}$ with the moving Lorentzian Darboux frame $\{\mathbf{T}, \boldsymbol{\eta}, \boldsymbol{\xi}\}$. Then the $\mathbf{T} \boldsymbol{\eta}$ Smarandache curve $\beta$ is spacelike and the Sabban frame $\left\{\beta, \mathbf{T}_{\beta}, \boldsymbol{\xi}_{\beta}\right\}$ is given by

$$
\begin{aligned}
& {\left[\begin{array}{c}
\beta \\
\mathbf{T}_{\beta} \\
\boldsymbol{\xi}_{\beta}
\end{array}\right]} \\
& =\left[\begin{array}{ccc}
\frac{a}{\sqrt{2}} & \frac{b}{\sqrt{2}} & 0 \\
\frac{b k_{n}}{\sqrt{\left(a k_{g}\right)^{2}-2 k_{n}^{2}}} & \frac{a k_{n}}{\sqrt{\left(a k_{g}\right)^{2}-2 k_{n}^{2}}} & \frac{a k_{g}}{\sqrt{\left(a k_{g}\right)^{2}-2 k_{n}^{2}}} \\
\frac{a b k_{g}}{\sqrt{2\left(a k_{g}\right)^{2}-4 k_{n}^{2}}} & \frac{a^{2} k_{g}}{\sqrt{2\left(a k_{g}\right)^{2}-4 k_{n}^{2}}} & \frac{-2 k_{n}}{\sqrt{2\left(a k_{g}\right)^{2}-4 k_{n}^{2}}}
\end{array}\right] \\
& \times\left[\begin{array}{c}
\mathbf{T} \\
\boldsymbol{\eta} \\
\xi
\end{array}\right],
\end{aligned}
$$

and the geodesic curvature $\bar{k}_{g}$ of the curve $\beta$ reads

$$
\bar{k}_{g}=\frac{a b k_{g} g_{1}-a^{2} k_{g} g_{2}-2 k_{n} g_{3}}{\sqrt{2}\left(a^{2} k_{g}^{2}-2 k_{n}^{2}\right)^{2}}
$$


where

$$
\begin{aligned}
\frac{d s^{*}}{d s}= & \sqrt{\frac{\left(a k_{g}\right)^{2}-2 k_{n}^{2}}{2}}, \\
g_{1}= & b a^{2} k_{g} k_{n} k_{g}^{\prime}-b a^{2} k_{g}^{2} k_{n}^{\prime}+a^{3} k_{g}^{4} \\
& -\left(a^{3}+2 a\right) k_{g}^{2} k_{n}^{2}+2 a k_{n}^{4}, \\
g_{2}= & -a^{3} k_{g}^{2} k_{n}^{\prime}+a^{3} k_{g} k_{n} k_{g}^{\prime}-b a^{2} k_{g}^{2} k_{n}^{2}+2 b k_{n}^{4}, \\
g_{3}= & 2 a k_{n}^{2} k_{g}^{\prime}-2 a k_{g} k_{n} k_{n}^{\prime}-b a^{2} k_{g}^{3} k_{n}+2 b k_{g} k_{n}^{3} .
\end{aligned}
$$

Proof. We assume that the curve $\alpha$ is a principal curve. Differentiating (15) with respect to $s$ and using (5) we obtain

$$
\begin{aligned}
\beta^{\prime} & =\frac{d \beta}{d s} \\
& =\frac{1}{\sqrt{2}}\left(b k_{n} \mathbf{T}+a k_{n} \boldsymbol{\eta}+a k_{g} \xi\right), \\
& \left\langle\beta^{\prime}, \beta^{\prime}\right\rangle=\frac{\left(a k_{g}\right)^{2}-2 k_{n}^{2}}{2},
\end{aligned}
$$

where from (9) $\left(a k_{g}\right)^{2}-2 k_{n}^{2}>0$ for all $s$. Since $\beta^{\prime}=$ $\left(d \beta / d s^{*}\right)\left(d s^{*} / d s\right)$, the tangent vector $\mathbf{T}_{\beta}$ of the curve $\beta$ is a spacelike vector such that

$$
\mathbf{T}_{\beta}=\frac{1}{\sqrt{\left(a k_{g}\right)^{2}-2 k_{n}^{2}}}\left(b k_{n} \mathbf{T}+a k_{n} \boldsymbol{\eta}+a k_{g} \xi\right),
$$

where

$$
\frac{d s^{*}}{d s}=\sqrt{\frac{\left(a k_{g}\right)^{2}-2 k_{n}^{2}}{2}} .
$$

On the other hand, from (15) and (40) it can be easily seen that

$$
\begin{aligned}
\xi_{\beta} & =-\beta \times T_{\beta} \\
& =\frac{1}{\sqrt{2\left(a k_{g}\right)^{2}-4 k_{n}^{2}}}\left(a b k_{g} \mathbf{T}+a^{2} k_{g} \boldsymbol{\eta}-2 k_{n} \xi\right)
\end{aligned}
$$

is a unit timelike vector.

Consequently, the geodesic curvature $\bar{k}_{g}$ of the curve $\beta=$ $\beta\left(s^{*}\right)$ is given by

$$
\begin{aligned}
\bar{k}_{g} & =\operatorname{det}\left(\beta, T_{\beta}, \mathbf{T}_{\beta}^{\prime}\right) \\
& =\frac{a b k_{g} g_{1}-a^{2} k_{g} g_{2}-2 k_{n} g_{3}}{\sqrt{2}\left(a^{2} k_{g}^{2}-2 k_{n}^{2}\right)^{2}},
\end{aligned}
$$

where

$$
\begin{aligned}
g_{1}= & b a^{2} k_{g} k_{n} k_{g}^{\prime}-b a^{2} k_{g}^{2} k_{n}^{\prime}+a^{3} k_{g}^{4} \\
& -\left(a^{3}+2 a\right) k_{g}^{2} k_{n}^{2}+2 a k_{n}^{4},
\end{aligned}
$$

$$
\begin{aligned}
& g_{2}=-a^{3} k_{g}^{2} k_{n}^{\prime}+a^{3} k_{g} k_{n} k_{g}^{\prime}-b a^{2} k_{g}^{2} k_{n}^{2}+2 b k_{n}^{4}, \\
& g_{3}=2 a k_{n}^{2} k_{g}^{\prime}-2 a k_{g} k_{n} k_{n}^{\prime}-b a^{2} k_{g}^{3} k_{n}+2 b k_{g} k_{n}^{3} .
\end{aligned}
$$

From (15), (40), and (42) we obtain the Sabban frame $\left\{\beta, \mathbf{T}_{\beta}, \boldsymbol{\xi}_{\beta}\right\}$ of $\beta$.

In the theorems which follow, in a similar way as in Theorem 12, we obtain the Sabban frame $\left\{\beta, \mathbf{T}_{\beta}, \boldsymbol{\xi}_{\beta}\right\}$ and the geodesic curvature $\bar{k}_{g}$ of a spacelike Smarandache curve. We omit the proofs of Theorems 13 and 15, since they are analogous to the proof of Theorem 12.

Theorem 13. Let $\alpha=\alpha(s)$ be a principal spacelike curve lying fully on the spacelike surface $M$ in $\mathbb{R}_{1}^{3}$ with the moving Lorentzian Darboux frame $\{\mathbf{T}, \boldsymbol{\eta}, \boldsymbol{\xi}\}$. Then the $\mathbf{T} \boldsymbol{\xi}$ Smarandache curve $\beta$ is spacelike and the Sabban frame $\left\{\beta, \mathbf{T}_{\beta}, \boldsymbol{\xi}_{\beta}\right\}$ is given by

$\left[\begin{array}{c}\beta \\ \mathbf{T}_{\beta} \\ \boldsymbol{\xi}_{\beta}\end{array}\right]$

$$
\begin{aligned}
& =\left[\begin{array}{ccc}
\frac{a}{\sqrt{2}} & 0 & \frac{b}{\sqrt{2}} \\
\frac{-b k_{g}}{\sqrt{2 k_{g}^{2}-\left(a k_{n}\right)^{2}}} & \frac{a k_{n}}{\sqrt{2 k_{g}^{2}-\left(a k_{n}\right)^{2}}} & \frac{a k_{g}}{\sqrt{2 k_{g}^{2}-\left(a k_{n}\right)^{2}}} \\
\frac{-a b k_{n}}{\sqrt{4 k_{g}^{2}-2\left(a k_{n}\right)^{2}}} & \frac{2 k_{g}}{\sqrt{4 k_{g}^{2}-2\left(a k_{n}\right)^{2}}} & \frac{-a^{2} k_{n}}{\sqrt{4 k_{g}^{2}-2\left(a k_{n}\right)^{2}}}
\end{array}\right] \\
& \times\left[\begin{array}{c}
\mathrm{T} \\
\boldsymbol{\eta} \\
\xi
\end{array}\right],
\end{aligned}
$$

and the geodesic curvature $\bar{k}_{g}$ of the curve $\beta$ reads

$$
\bar{k}_{g}=-\frac{a b k_{n} h_{1}+2 k_{g} h_{2}+a^{2} k_{n} h_{3}}{\sqrt{2}\left(2 k_{g}^{2}-\left(a k_{n}\right)^{2}\right)^{2}},
$$

where

$$
\begin{gathered}
\frac{d s^{*}}{d s}=\sqrt{\frac{2 k_{g}^{2}-\left(a k_{n}\right)^{2}}{2}}, \\
h_{1}=-b a^{2} k_{n}^{2} k_{g}^{\prime}+b a^{2} k_{g} k_{n} k_{n}^{\prime}+2 a k_{g}^{4} \\
-a^{3} k_{g}^{2} k_{n}^{2}-2 a k_{g}^{2} k_{n}^{2}+a^{3} k_{n}^{4}, \\
h_{2}=2 a k_{g} k_{n} k_{g}^{\prime}-2 a k_{g}^{2} k_{n}^{\prime}-2 b k_{g}^{3} k_{n}+b a^{2} k_{g} k_{n}^{3}, \\
h_{3}=a^{3} k_{n}^{2} k_{g}^{\prime}-a^{3} k_{g} k_{n} k_{n}^{\prime}-2 b k_{g}^{4}+b a^{2} k_{g}^{2} k_{n}^{2} .
\end{gathered}
$$

Theorem 14. Let $\alpha=\alpha(s)$ be a principal spacelike curve lying fully on the spacelike surface $M$ in $\mathbb{R}_{1}^{3}$ with the moving Lorentzian Darboux frame $\{\mathbf{T}, \boldsymbol{\eta}, \boldsymbol{\xi}\}$. Then 
(i) if $a k_{n}-b k_{g} \neq 0$ for all $s$, the $\boldsymbol{\eta} \xi$-Smarandache curve $\beta$ is spacelike and the Sabban frame $\left\{\beta, \mathbf{T}_{\beta}, \boldsymbol{\xi}_{\beta}\right\}$ is given by

$$
\left[\begin{array}{c}
\beta \\
\mathbf{T}_{\beta} \\
\boldsymbol{\xi}_{\beta}
\end{array}\right]=\left[\begin{array}{ccc}
0 & \frac{a}{\sqrt{2}} & \frac{b}{\sqrt{2}} \\
\epsilon & 0 & 0 \\
0 & -\epsilon \frac{b}{\sqrt{2}} & \epsilon \frac{a}{\sqrt{2}}
\end{array}\right]\left[\begin{array}{c}
\mathbf{T} \\
\boldsymbol{\eta} \\
\boldsymbol{\xi}
\end{array}\right],
$$

and the geodesic curvature $\bar{k}_{g}$ of the curve $\beta$ reads

$$
\bar{k}_{g}=-\frac{a k_{g}+b k_{n}}{\sqrt{2}},
$$

where $\epsilon=\operatorname{sign}\left(a k_{n}-b k_{g}\right)$ for all s and

$$
\frac{d s^{*}}{d s}=\epsilon \frac{a k_{n}-b k_{g}}{\sqrt{2}}
$$

(ii) if $a k_{n}-b k_{g}=0$ for all $s$, then the Sabban frame $\left\{\beta, \mathbf{T}_{\beta}, \boldsymbol{\xi}_{\beta}\right\}$ of the $\boldsymbol{\eta} \boldsymbol{\xi}$-Smarandache curve $\beta$ is a null geodesic.

Proof. We assume that the curve $\alpha$ is a principal curve. Differentiating (17) with respect to $s$ and using (5) we obtain

$$
\begin{aligned}
\beta^{\prime} & =\frac{d \beta}{d s^{*}} \frac{d s^{*}}{d s} \\
& =\frac{1}{\sqrt{2}}\left(a k_{n}-b k_{g}\right) \mathrm{T}, \\
\left\langle\beta^{\prime}, \beta^{\prime}\right\rangle & =\frac{\left(a k_{n}-b k_{g}\right)^{2}}{2} .
\end{aligned}
$$

Then, there are two following cases.

(i) If $a k_{n}-b k_{g} \neq 0$ for all $s$, since $\beta^{\prime}=\left(d \beta / d s^{*}\right)\left(d s^{*} / d s\right)$, then we obtain that the unit tangent vector $\mathbf{T}_{\beta}$ of the curve $\beta$ is a spacelike vector such that

$$
\mathbf{T}_{\beta}=\epsilon \mathbf{T},
$$

where

$$
\frac{d s^{*}}{d s}=\epsilon \frac{a k_{n}-b k_{g}}{\sqrt{2}}
$$

and $\epsilon=\operatorname{sign}\left(a k_{n}-b k_{g}\right)$.

On the other hand, from (17) and (52) it can be easily seen that

$$
\begin{aligned}
\xi_{\beta} & =-\beta \times T_{\beta} \\
& =-\epsilon \frac{b}{\sqrt{2}} \boldsymbol{\eta}+\epsilon \frac{a}{\sqrt{2}} \boldsymbol{\xi}
\end{aligned}
$$

is a unit timelike vector.
Consequently, the geodesic curvature $\bar{k}_{g}$ of the curve $\beta=\beta\left(s^{*}\right)$ is given by

$$
\begin{aligned}
\bar{k}_{g} & =\operatorname{det}\left(\beta, T_{\beta}, \mathbf{T}_{\beta}^{\prime}\right) \\
& =-\frac{a k_{g}+b k_{n}}{\sqrt{2}} .
\end{aligned}
$$

From (17), (52), and (54) we obtain the Sabban frame $\left\{\beta, \mathbf{T}_{\beta}, \boldsymbol{\xi}_{\beta}\right\}$ of $\beta$.

(ii) If $a k_{n}-b k_{g}=0$ for all $s$, then $\beta^{\prime}$ is null. So, the tangent vector $\mathbf{T}_{\beta}$ of the curve $\beta$ is a null vector. It is known that the only null curves lying on pseudosphere $S_{1}^{2}$ are the null straight lines, which are the null geodesics.

Theorem 15. Let $\alpha=\alpha(s)$ be a principal spacelike curve lying fully on the spacelike surface $M$ in $\mathbb{R}_{1}^{3}$ with the moving Lorentzian Darboux frame $\{\mathbf{T}, \boldsymbol{\eta}, \boldsymbol{\xi}\}$. Then the $\mathrm{T} \eta \xi$-Smarandache curve $\beta$ is spacelike and the Sabban frame $\left\{\beta, \mathbf{T}_{\beta}, \boldsymbol{\xi}_{\beta}\right\}$ is given by

$$
\begin{gathered}
\beta=\frac{1}{\sqrt{3}}(a \mathbf{T}+b \boldsymbol{\eta}+c \boldsymbol{\xi}), \\
\mathbf{T}_{\beta}=\frac{\left(b k_{n}-c k_{g}\right) \mathbf{T}+a k_{n} \boldsymbol{\eta}+a k_{g} \xi}{\sqrt{3\left(k_{n}^{2}-k_{g}^{2}\right)+\left(b k_{g}-c k_{n}\right)^{2}}} \\
\xi_{\beta}=\left(\left(a b k_{g}-a c k_{n}\right) \mathbf{T}+\left(\left(3+b^{2}\right) k_{g}-b c k_{n}\right) \boldsymbol{\eta}\right. \\
\left.-\left(\left(3-c^{2}\right) k_{n}+b c k_{g}\right) \boldsymbol{\xi}\right) \\
\times\left(\sqrt{9\left(k_{n}^{2}-k_{g}^{2}\right)+3\left(b k_{g}-c k_{n}\right)^{2}}\right)^{-1},
\end{gathered}
$$

and the geodesic curvature $\bar{k}_{g}$ of the curve $\beta$ reads

$$
\begin{aligned}
\bar{k}_{g}= & -\left(\left(a b k_{g}-a c k_{n}\right) l_{1}-\left(\left(3+b^{2}\right) k_{g}-b c k_{n}\right) l_{2}\right. \\
& \left.-\left(\left(3-c^{2}\right) k_{n}+b c k_{g}\right) l_{3}\right) \\
& \times\left(\sqrt{2}\left(a^{2} k_{g}^{2}-2 k_{n}^{2}\right)^{2}\right)^{-1},
\end{aligned}
$$

where

$$
\begin{gathered}
\frac{d s^{*}}{d s}=\frac{\sqrt{3\left(k_{n}^{2}-k_{g}^{2}\right)+\left(b k_{g}-c k_{n}\right)^{2}}}{\sqrt{3}}, \\
l_{1}=\left(3 a b k_{n}^{2}-3 a c k_{g} k_{n}\right) k_{g}^{\prime}+\left(3 a c k_{g}^{2}-3 a b k_{g} k_{n}\right) k_{n}^{\prime} \\
+\left(b k_{n}-c k_{g}\right)\left(b k_{g}-c k_{n}\right)^{3}+3 b c k_{g}^{4}-3 b c k_{n}^{4} \\
+3\left(b^{2}+c^{2}\right) k_{g} k_{n}^{3}-3\left(b^{2}+c^{2}\right) k_{g}^{3} k_{n},
\end{gathered}
$$




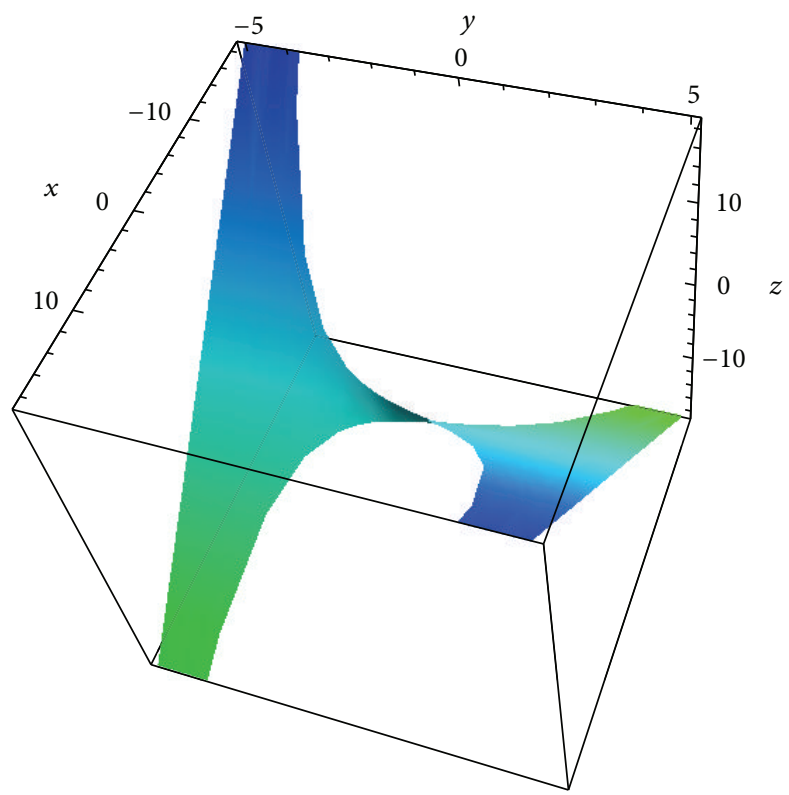

FIGURE 1: The spacelike surface $\phi(s, u)$.

$$
\begin{aligned}
l_{2}= & \left(\left(3 b^{2}+3 c^{2}+9\right) k_{n}^{2}-6 b c k_{g} k_{n}\right) k_{g}^{\prime} \\
& -\left(\left(3 b^{2}+3 c^{2}+9\right) k_{g} k_{n}-6 b c k_{g}^{2}\right) k_{n}^{\prime} \\
& -\left(a c^{3}+3 a c\right) k_{n}^{4}+\left(3 a b+3 a b c^{2}\right) k_{g} k_{n}^{3} \\
& +\left(a b^{3}-3 a b\right) k_{g}^{3} k_{n}+\left(3 a c-3 a b^{2} c\right) k_{g}^{2} k_{n}^{2}, \\
l_{3}= & \left(\left(3 b^{2}+3 c^{2}-9\right) k_{g} k_{n}-6 b c k_{n}^{2}\right) k_{g}^{\prime} \\
& +\left(\left(9-3 b^{2}-3 c^{2}\right) k_{g}^{2}+6 b c k_{g} k_{n}\right) k_{n}^{\prime} \\
& +\left(a b^{3}-3 a b\right) k_{g}^{4}-\left(3 a c+a c^{3}\right) k_{g} k_{n}^{3} \\
& +\left(3 a c-3 a b^{2} c\right) k_{g}^{3} k_{n}+\left(3 a b+3 a b c^{2}\right) k_{g}^{2} k_{n}^{2} .
\end{aligned}
$$

Example 16. Let us define a spacelike ruled surface (see Figure 1) in the Minkowski 3-space such as

$$
\begin{gathered}
\phi: U \subset \mathbb{R}^{2} \longrightarrow \mathbb{R}_{1}^{3} \\
(s, u) \longrightarrow \phi(s, u)=\alpha(s)+u \mathbf{e}(s), \\
\phi(s, u)=(-u \sinh s, s,-u \cosh s),
\end{gathered}
$$

where $u \in(-1,1)$.
Then we get the Lorentzian Darboux frame $\{\mathbf{T}, \boldsymbol{\eta}, \boldsymbol{\xi}\}$ along the curve $\alpha$ as follows:

$$
\begin{gathered}
\mathbf{T}(s)=(0,1,0), \\
\boldsymbol{\eta}(s) \equiv \frac{1}{\sqrt{1-u^{2}}}(\cosh s,-u, \sinh s), \\
\xi(s)=\frac{1}{\sqrt{1-u^{2}}}(-\sinh s, 0,-\cosh s),
\end{gathered}
$$

where $\boldsymbol{\xi}(s)$ is spacelike vectors and $\boldsymbol{\eta}(s)$ is a unit timelike vector.

Moreover, the geodesic curvature $k_{g}(s)$, the asymptotic curvature $k_{n}(s)$, and the principal curvature $\tau_{g}(s)$ of the curve $\alpha$ have the form

$$
\begin{gathered}
k_{g}(s)=\left\langle\mathbf{T}^{\prime}(s), \boldsymbol{\xi}(s)\right\rangle=0, \\
k_{n}(s)=-\left\langle\mathbf{T}^{\prime}(s), \boldsymbol{\eta}(s)\right\rangle=0, \\
\tau_{g}(s)=-\left\langle\boldsymbol{\xi}^{\prime}(s), \boldsymbol{\eta}(s)\right\rangle=-\frac{1}{1-u^{2}} .
\end{gathered}
$$

Taking $a=\sqrt{3}, b=1$ and using (15), we obtain that the $\mathrm{T} \boldsymbol{\eta}$-Smarandache curve $\beta$ of the curve $\alpha$ is given by (see Figure 2(a))

$$
\beta\left(s^{\star}(s)\right)=\left(\frac{\cosh s}{\sqrt{1-u^{2}}}, \sqrt{3}-\frac{u}{\sqrt{1-u^{2}}}, \frac{\sinh s}{\sqrt{1-u^{2}}}\right) .
$$

Taking $a=b=1$ and using (16), we obtain that the $\mathbf{T} \xi$-Smarandache curve $\beta$ of the curve $\alpha$ is given by (see Figure 2(b))

$$
\beta\left(s^{\star}(s)\right)=\left(-\frac{\sinh s}{\sqrt{1-u^{2}}}, 1,-\frac{\cosh s}{\sqrt{1-u^{2}}}\right) .
$$




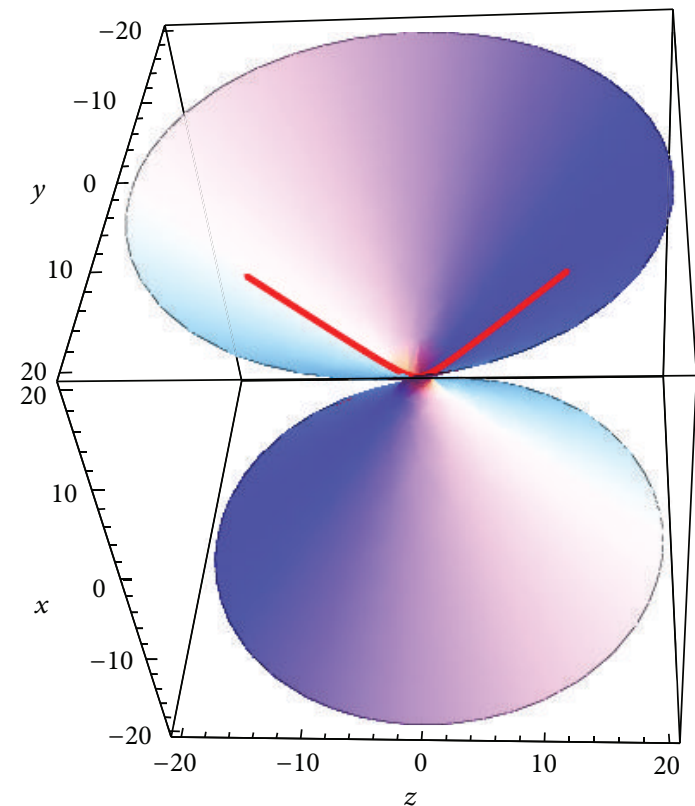

(a)

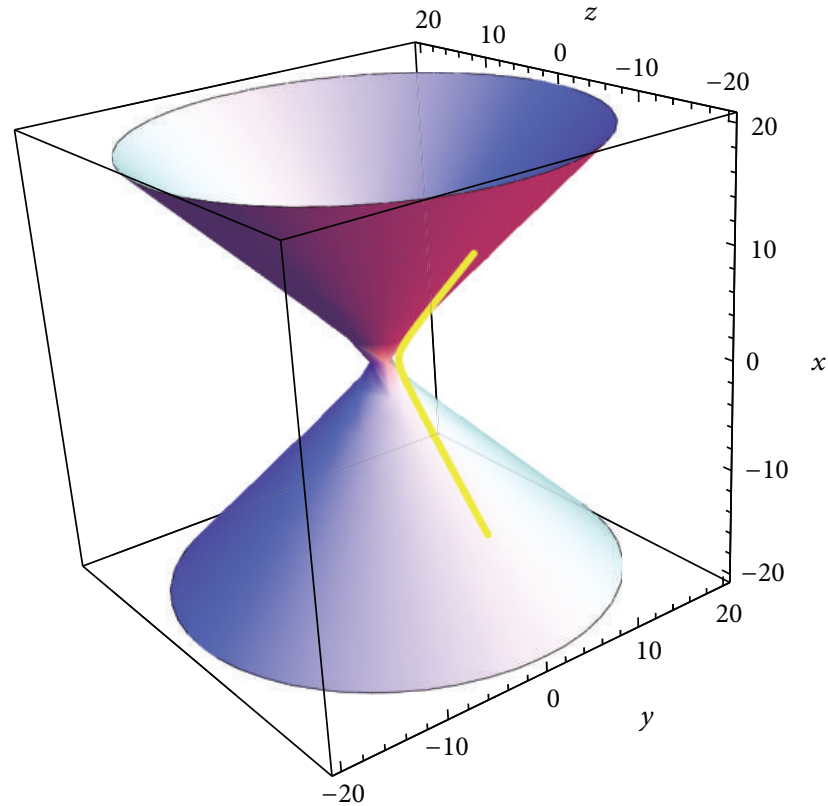

(b)

Figure 2: (a) The $\mathbf{T} \boldsymbol{\eta}$-Smarandache curve $\beta$ on $S_{1}^{2}$ for $u=1 / \sqrt{3}$. (b) The $\mathbf{T} \boldsymbol{\xi}$-Smarandache curve $\beta$ on $S_{1}^{2}$ for $u=1 / \sqrt{3}$.

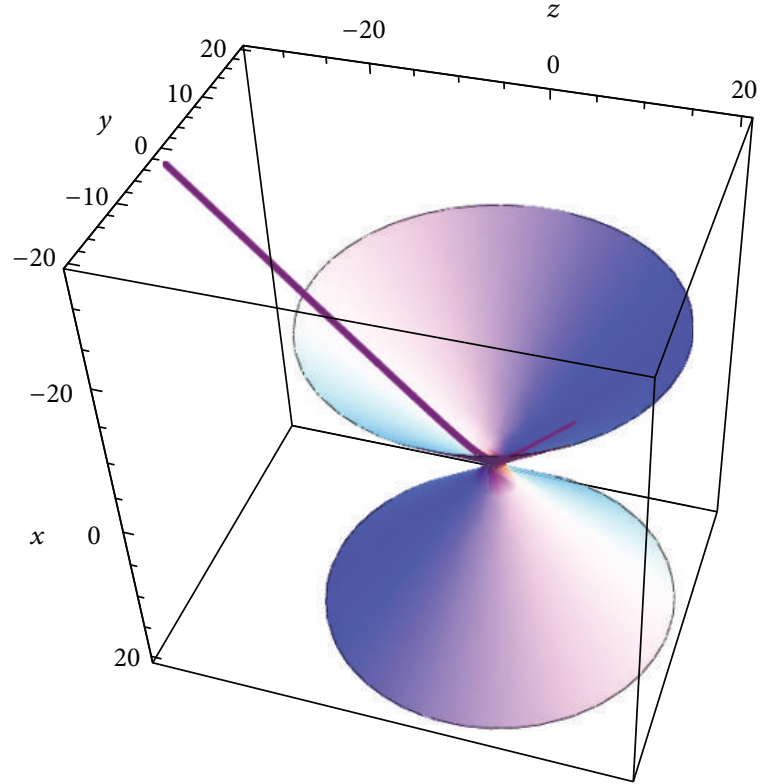

(a)

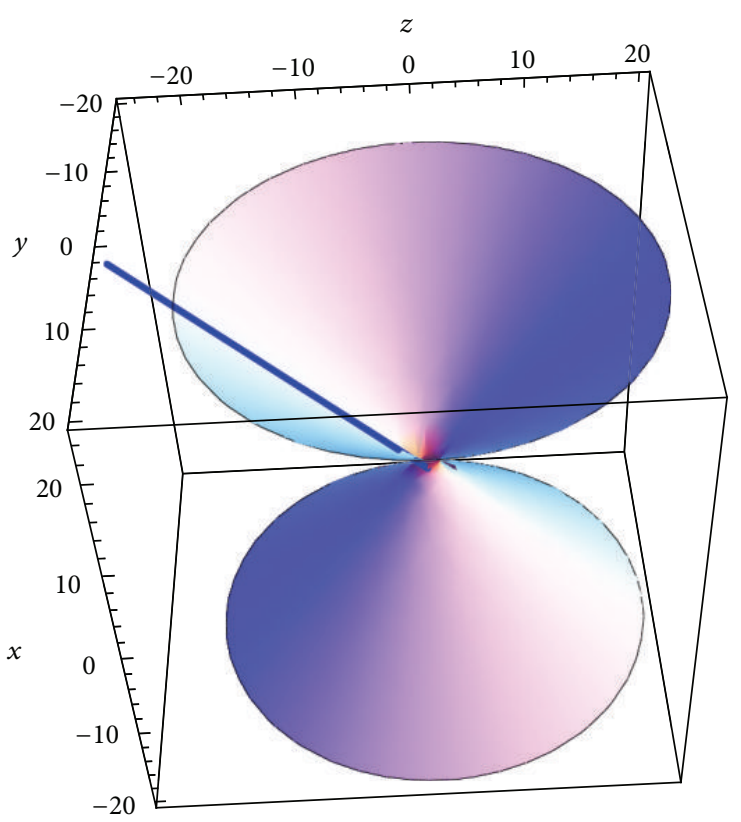

(b)

Figure 3: (a) The $\boldsymbol{\eta} \xi$-Smarandache curve $\beta$ on $S_{1}^{2}$ for $u=1 / \sqrt{3}$. (b) The T $\boldsymbol{\eta} \xi$-Smarandache curve $\beta$ on $S_{1}^{2}$ for $u=1 / \sqrt{3}$.

Taking $a=\sqrt{3}, b=1$ and using (17), we obtain that the $\eta \xi$-Smarandache curve $\beta$ of the curve $\alpha$ is given by (see Figure 3(a))

$$
\begin{aligned}
\beta\left(s^{\star}(s)\right)= & -\frac{1}{\sqrt{1-u^{2}}} \\
& \times(\sinh s-\sqrt{3} \cosh s, \sqrt{3} u, \cosh s-\sqrt{3} \sinh s) .
\end{aligned}
$$

Taking $a=\sqrt{3}, b=1$, and $c=1$ and using (18), we obtain that the $\mathbf{T} \eta \xi$-Smarandache curve $\beta$ of the curve $\alpha$ is given by (see Figure 3(b))

$$
\begin{aligned}
\beta\left(s^{\star}(s)\right)= & \frac{1}{\sqrt{1-u^{2}}} \\
& \times(\cosh s-\sinh s, \\
& \left.\quad \sqrt{3-3 u^{2}}-u, \sinh s-\cosh s\right) .
\end{aligned}
$$




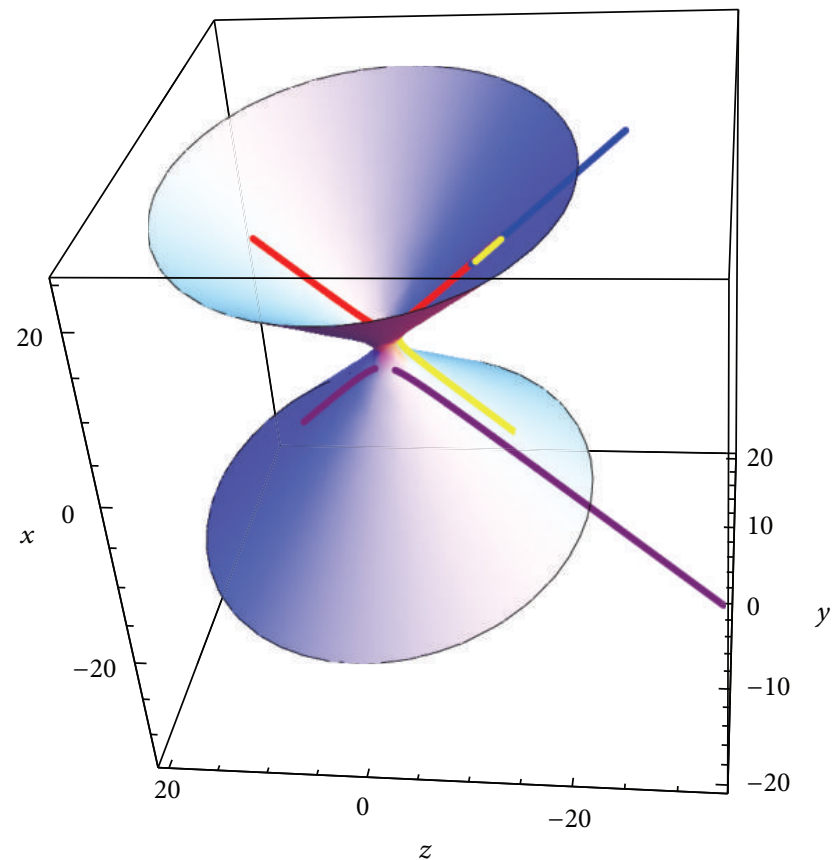

FIgURE 4: The Smarandache curves on $S_{1}^{2}$ for $u=1 / \sqrt{3}$.

Also, the Smarandache curves on $S^{2}$ of $\alpha$ for $u=1 / \sqrt{3}$ with Figure 4 are shown.

\section{Conflict of Interests}

The authors declare that there is no conflict of interests regarding the publication of this paper.

\section{References}

[1] C. Ashbacher, "Smarandache geometries," Smarandache Notions Journal, vol. 8, no. 1-3, pp. 212-215, 1997.

[2] M. Turgut and S. Yilmaz, "Smarandache curves in Minkowski space-time," International Journal of Mathematical Combinatorics, vol. 3, pp. 51-55, 2008.

[3] A. T. Ali, "Special smarandache curves in the euclidean space," International Journal of Mathematical Combinatorics, vol. 2, pp. 30-36, 2010.

[4] E. B. Koc Ozturk, U. Ozturk, K. İlarslan, and E. Nešović, "On pseudohyperbolical Smarandache curves in Minkowski 3space," International Journal of Mathematics and Mathematical Sciences, vol. 2013, Article ID 658670, 7 pages, 2013.

[5] E. B. Koc Ozturk, U. Ozturk, K. Ilarslan, and E. Nešović, "On pseudospherical smarandache curves in Minkowski 3-space," Journal of Applied Mathematics, vol. 2014, Article ID 404521, 14 pages, 2014.

[6] K. Taşköprü and M. Tosun, "Smarandache curves on $S^{2}$," Boletim da Sociedade Paranaense de Matemática, vol. 32, no. 1, pp. 51-59, 2014.

[7] T. Korpinar and E. Turhan, "A new approach on Smarandache TN-curves in terms of spacelike biharmonic curves with a timelike binormal in the Lorentzian Heisenberg group $\mathrm{Heis}^{3}$," Journal of Vectorial Relativity, vol. 6, pp. 8-15, 2011.
[8] T. Korpinar and E. Turhan, "Characterization of Smarandache $M_{1} M_{2}$-curves of spacelike biharmonic $B$-slant helices according to Bishop frame in $\mathbb{E}(1,1)$," Advanced Modeling and Optimization, vol. 14, no. 2, pp. 327-333, 2012.

[9] O. Bektas and S. Yuce, "Special Smarandache Curves According to Darboux Frame in $\mathrm{E}^{3}$," Romanian Journal of Mathematics and Computer Science, vol. 3, no. 1, pp. 48-59, 2013.

[10] B. O'Neill, Semi-Riemannian Geometry with Applications to Relativity, Academic Press, New York, NY, USA, 1983. 


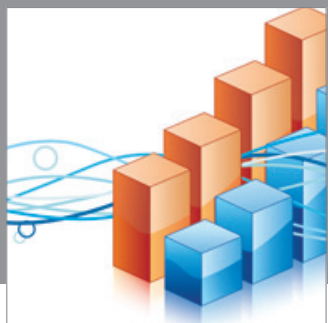

Advances in

Operations Research

mansans

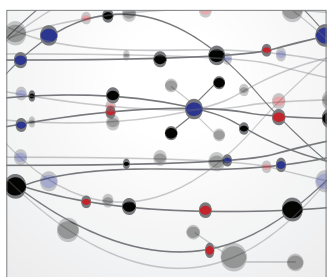

The Scientific World Journal
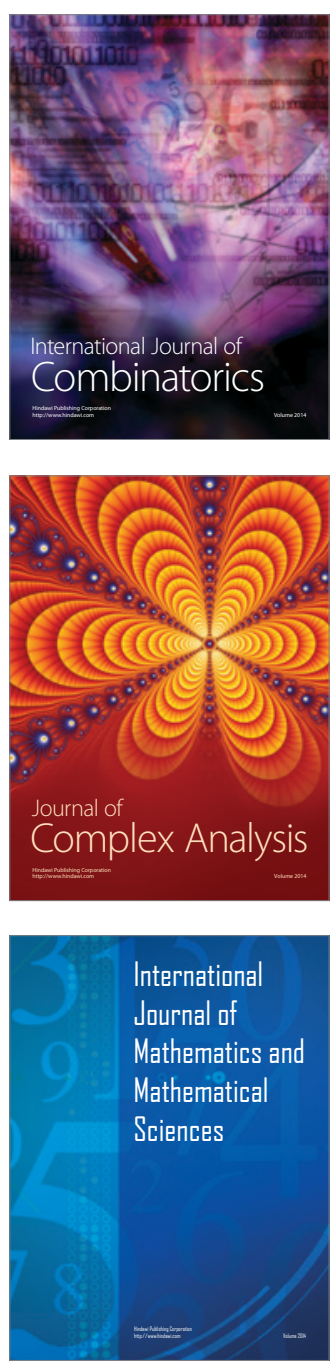
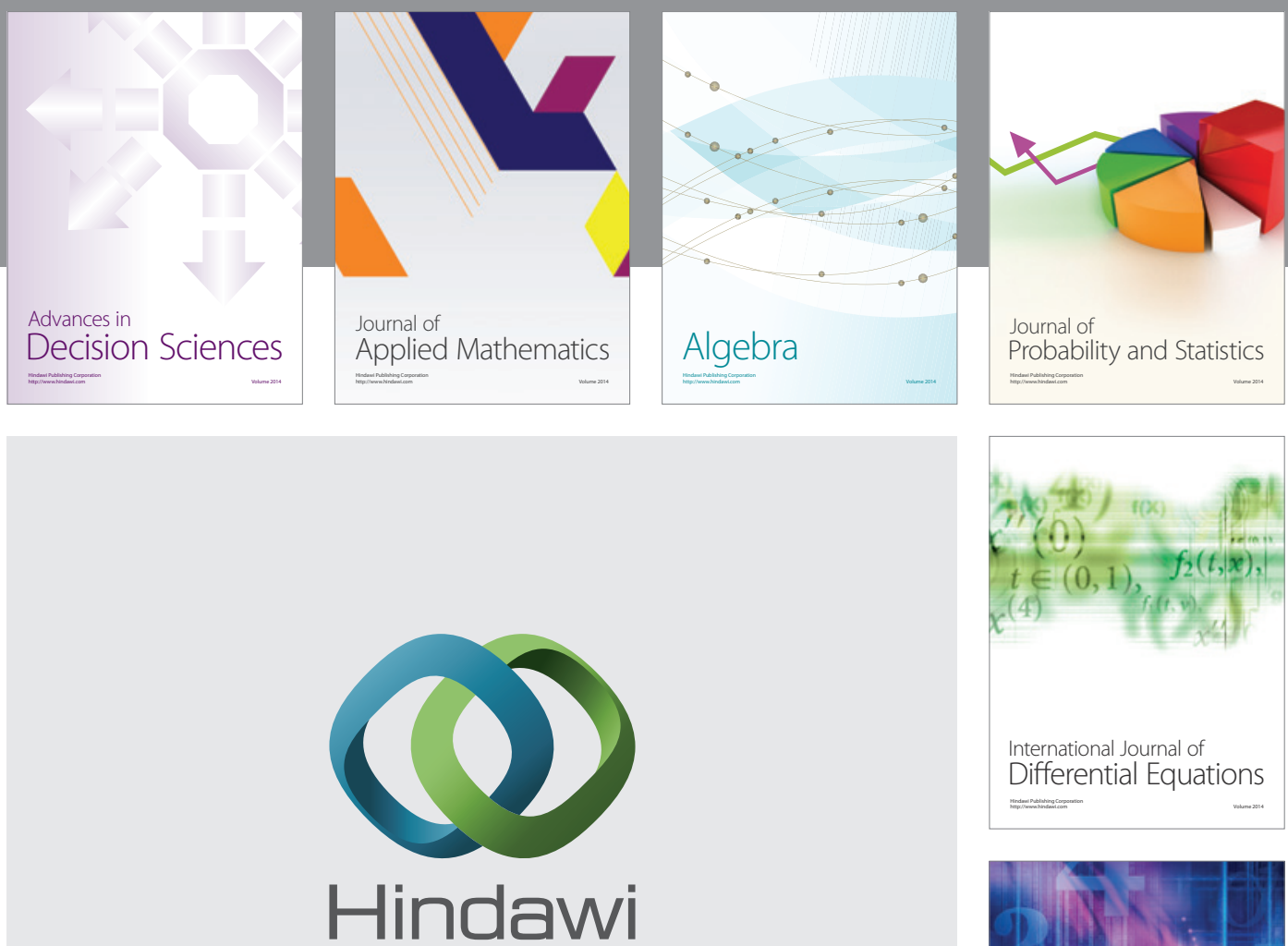

Submit your manuscripts at http://www.hindawi.com
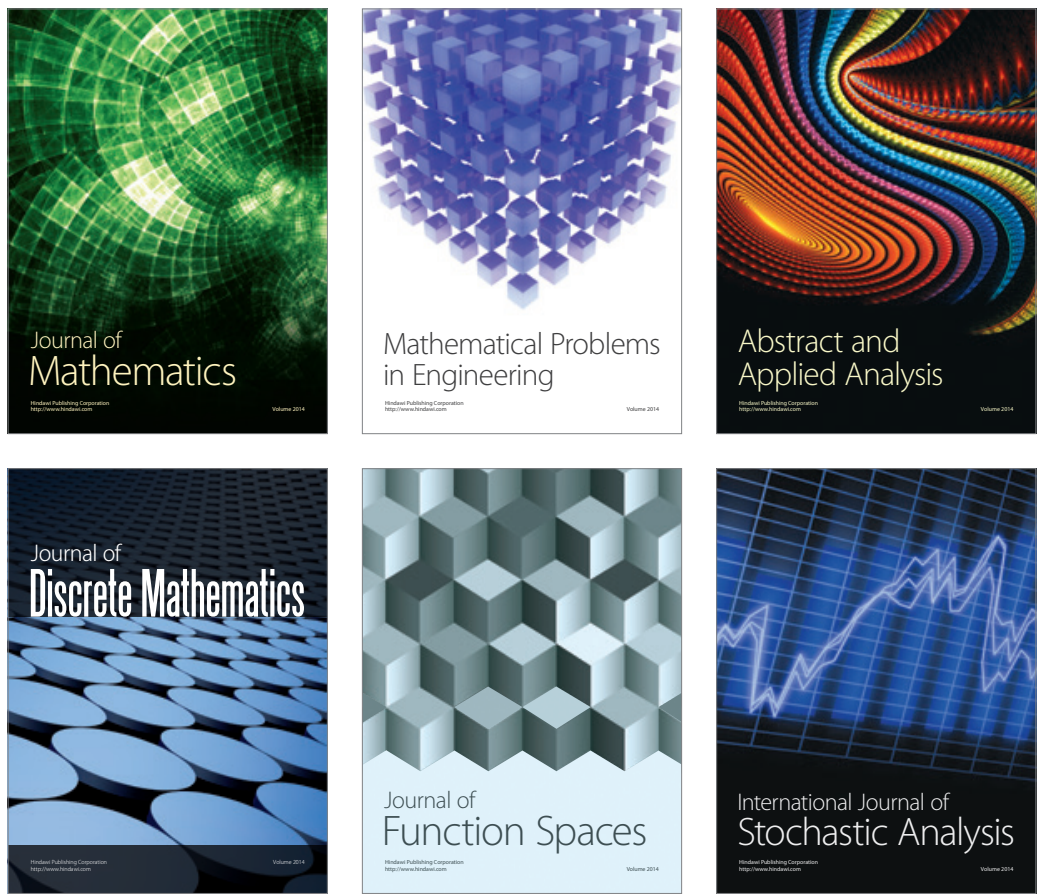

Journal of

Function Spaces

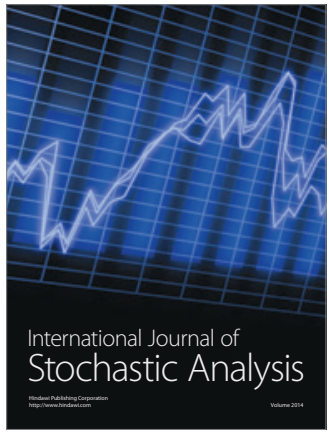

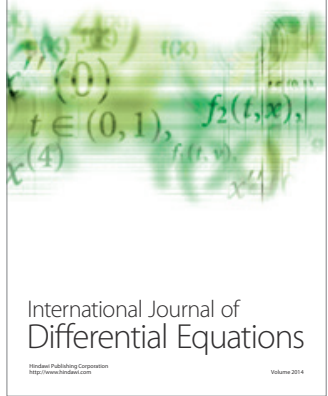
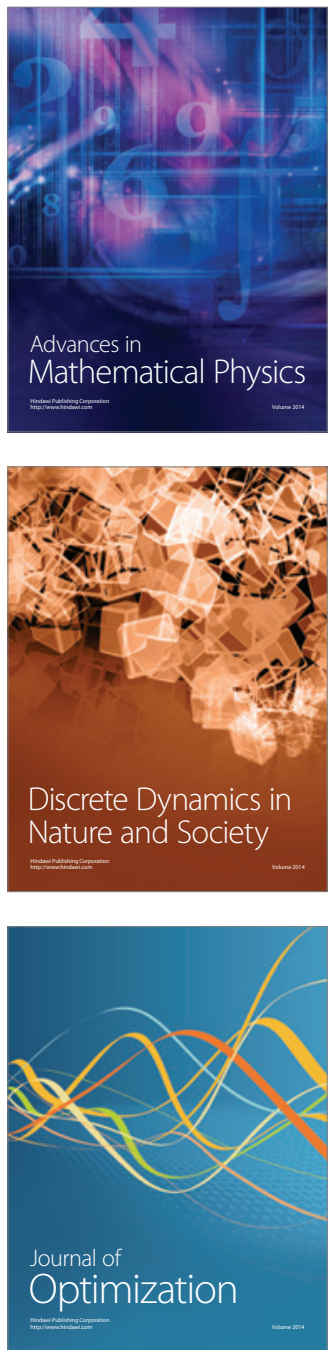